\title{
Availability of related long-term memory during and after attention focus in working memory
}

\author{
DAN J. WOLTZ \\ University of Utah, Salt Lake City, Utah \\ and \\ CHRISTOPHER A. WAS \\ Kent State University, Kent, Ohio
}

\begin{abstract}
In a series of four experiments, we investigated the magnitude and pattern of indirect, semantically mediated priming that emanates from various working memory (WM) processes. When such priming effects are taken to reflect temporary increases in the availability of long-term memory (ALTM), results suggest a close link between the amount and type of attention-driven processing in WM and the resulting accessibility of semantically related memory structures. Patterns of priming were equivalent when attention demands were prior to, versus concurrent with, the priming measures, suggesting that ALTM processes either require no additional cognitive resources or require resources that are independent of those underlying effortful WM processes. The results are discussed with respect to emerging evidence for long-term semantic priming and models of WM that incorporate active but unattended information as part of a limited capacity cognitive workspace.
\end{abstract}

The construct of working memory (WM), as described by Baddeley and Hitch (1974), has had a far-reaching impact on theories of cognition. For years, Baddeley's model was the predominant theory of WM, and it remained relatively unchanged, with its two storage components for visuospatial and phonological information and a central executive component for coordinating storage and various processing functions. ${ }^{1} \mathrm{~A}$ defining feature of all three of these subcomponents has been their attention-driven nature. In fact, at one point, Baddeley (1993) reflected that it may have been more appropriate to use the term working attention, rather than working memory. The term attention is used by Baddeley, as well as by many other authors within the WM literature, to refer broadly to effortful processes that range from rehearsal procedures to executive functions, such as planning, organizing, and controlling cognitive actions. In this article, we rely on the broad distinction between deliberate, attention-driven processes of WM that operate within the awareness of the individual and processes that operate outside of conscious awareness, such as implicit memory processes that underlie various forms of priming.

Various theorists have noted that relatively smallcapacity, attention-driven storage components of WM fail to explain complex cognitive activities such as language

Correspondence concerning this article should be addressed to D. J. Woltz, Department of Educational Psychology, University of Utah, 1705 East Campus Center Drive, Room 327, Salt Lake City, UT 84112-9255 (e-mail: woltz@ed.utah.edu).

Note-This article was accepted by the previous editorial team, when Colin M. MacLeod was Editor. comprehension, in which a great deal of information needs to be temporarily available for processing (e.g., Anderson, 1983; Cowan, 1999; Ericsson \& Kintsch, 1995; Just \& Carpenter, 1992; Kintsch, Patel, \& Ericsson, 1999). Corresponding to this criticism, alternative conceptualizations of WM have been proposed that allow for more information to be available for processing at any given moment. Most define this greater availability in terms of long-term memory (LTM) activation, or the temporary increase in retrieval strength of existing memory representations. These models differ in important respects, but of interest here is their common inclusion of long-term memory structures that are temporarily available for processing but that are not in the current focus of attention. Most of these alternative models postulate that both attentiondriven WM processes and automatic LTM activation or retrieval processes effectively define capacity limits that constrain complex processing activities. Furthermore, some suggest that the LTM activation processes are capacity limited in a different manner than the attention-driven processes. However, direct empirical evidence regarding these issues is limited.

\section{LTM Processes in WM Models}

A variety of WM models that represent alternatives to Baddeley's model have been proposed within different theoretical contexts (see Miyake \& Shah, 1999). We will describe only a few of these that are most prominent in their inclusion of active-but-unattended LTM elements in WM. Virtually all WM theorists acknowledge the contribution of LTM knowledge structures to WM performance, but some have proposed models in which LTM processes are central to the definition of WM. 
Several WM proposals emerging from production systems have defined WM simply as active elements of LTM. In an early description of the production system approach, Anderson (1983) noted that the inclusion of WM in system architecture was central but that capacity limits imposed on WM needed to be unconventional. He described efforts to simulate cognitive performance, citing informal proposals to increase the contents of WM to 20 or more elements, a figure that far exceeded the capacity limits estimated for the storage components of Baddeley's model of WM or previous short-term memory storage estimates (Baddeley, 1986; Baddeley \& Hitch, 1974). Broadbent (1993) arrived at a similar conclusion regarding computer simulations of complex cognitive activities. Consistent with this assessment, Anderson $(1983,1993)$ defined WM simply as the contents of long-term declarative memory that are currently active, with activation varying on a gradient, rather than reflecting all-or-nothing availability. There was no capacity limit per se, except that activation had to be shared by all the elements receiving activation from a given source. However, Anderson, Reder, and Lebiere (1996) added an explicit assumption that the amount of source activation had a fixed limit that reflected the amount of attention that could be devoted to source objects. Thus, the capacity for activation was linked to that of attention, despite the fact that much more information could be activated to some level of availability than would be expected from strict attention-based WM models.

Just and Carpenter (1992) have proposed a similar concept of WM within a production system model for language comprehension. They proposed that both storage and processing functions in WM were dependent on the same limited capacity resource of activation. Since the aim of their model was partly to explain individual differences in comprehension, they assumed that activation capacity had a fixed limit that varied across individuals (for a similar concept, see Cantor \& Engle, 1993). There was no explicit distinction between activation and attention resources. In fact, for much of the empirical evidence for their activation capacity model, the Daneman and Carpenter (1980) span task has been utilized, which frequently has been used as a measure of individual differences in attention-based WM.

Ericsson and Kintsch (1995) have proposed a model of WM that incorporates LTM but differs somewhat from the production system accounts. They distinguished between two components of WM: short-term WM (ST-WM) and long-term WM (LT-WM). ST-WM resembled Baddeley's WM concept and was implicated in the processing of novel tasks in which information must be actively maintained with attention-driven processes. In contrast, LT-WM described the ready access to well-learned knowledge structures, and it was implicated in performance within highly familiar tasks or knowledge domains. This WM model differs somewhat from those previously mentioned, in that the availability of LTM structures for processing is attributed to skilled retrieval structures that develop through extensive practice. Furthermore, there is no explicit capacity limit associated with LT-WM. Instead, it reflects domain-specific skills of automatically accessing related LTM elements.

Two additional WM models of interest are similar to that of Ericsson and Kintsch (1995) in proposing an explicit distinction between information that is in the focus of attention in WM and activated information in LTM that is available but unattended. Cowan's $(1995,1999)$ embedded process model specifies that a small amount of LTM information is in the focus of attention at any one time. A larger subset of LTM is activated due to prior attention focus and current perceptual processes, and this information is available to varying degrees for processing. Either automatic or attention-driven processes may operate on the active information. This model combines both activation and attention processes to define WM limits. Attention is seen to have limits as to the amount of information that can be in attentional focus at any given time. Activation is seen to have time limits (i.e., activated information loses strength with time if not reactivated). Cowan and colleagues have performed a variety of experiments that support various aspects of this conceptualization (see Cowan, 1995, 1999). However, the experimental work has emphasized the availability of information that was previously presented (e.g., spoken syllables or memory list items), rather than LTM contents related to what is being processed. Such evidence is important, but it does not represent the full range of LTM contents that must be available in WM during language comprehension and problem solving (i.e., related domain knowledge).

Oberauer (2002) has proposed a concentric model of WM that closely resembles that of Cowan (1999), in that information exists in different states of accessibility. Only one chunk of information is assumed to be directly in the focus of attention at any time. However, a limited number of additional chunks are assumed to be in a state of direct access, or at a level of activation at which the elements can readily become the focus of attention. Beyond information in a direct access state (which resembles an attention-based WM concept), there is a segment of LTM that holds some degree of increased accessibility, but its access would depend on retrieval structures such as those described by Ericsson and Kintsch (1995). Like Cowan $(1995,1999)$, Oberauer proposed that information held in a passive state of readiness (i.e., activated LTM) is not constrained by the same capacity limits as those that constrain information held in the focus of attention or in direct access to attention.

Oberauer (2002) reported results from two experiments that are consistent with his model. In these experiments, as in those by Cowan $(1995,1999)$, active information in LTM corresponded to stimulus content that had been processed previously but not actively maintained or manipulated. Again, this is an inadequate test of the broader notion that the cognitive workspace must include available background knowledge related to immediate task demands to explain complex forms of cognition, such as language comprehension and problem solving.

In sum, several alternative WM models to that of Baddeley (1986) have proposed a greater capacity for informa- 
tion that is available for processing in a general cognitive workspace. They do so by incorporating mechanisms that provide ready access to LTM elements that are not actively maintained by attention processes. In these models, the mechanisms vary between automatic activation processes and skilled retrieval structures that execute automatically. In addition, these models specify different functional relationships between available LTM elements and attention processes. Some make little or no distinction between the resources underlying attention and activation, whereas others propose distinct capacity limits for the two.

Empirical attempts at testing aspects of these models have demonstrated that information not in the focus of attention can be in different states of availability for processing. This conclusion is consistent with other research not specifically linked to a particular WM model (e.g., Haarmann, Davelaar, \& Usher, 2003; Potter, 1993). However, previous empirical research in this area has emphasized the availability of just-processed information and has failed to adequately address the availability of related knowledge structures in the context of a limited capacity workspace. This seems to be a serious limitation, given that theories of language comprehension have stressed the importance of constructive and integrative processes that connect new information to existing knowledge structures (e.g., Gernsbacher, 1990; Kintsch, 1998). In the research described here, this shortcoming was addressed.

In reference to memory contents that have heightened availability for processing but are not in the focus of attention, we use the term available long-term memory (ALTM), rather than other terms previously used in the literature. We do this to distinguish the construct we are investigating from others that reflect potentially different phenomena (e.g., such terms as LT-WM, short-term conceptual memory, or activated LTM). Of primary importance in the use of different terminology is that the memory processes we investigated involve the readiness of semantically related information in LTM, rather than just-processed information. In addition, we do not wish to constrain our interpretation of increased availability to either spreading activation or skilled retrieval mechanisms, as the use of some prior terminology would imply. We believe there may be a useful distinction between these mechanisms along the lines of declarative and procedural knowledge differences, and we hold open the possibility that both may operate to make related LTM more available.

\section{Overview of the Present Research}

The present research tested the fundamental premise that the content and complexity of attention-driven processing in WM determine the subsequent availability of semantically related elements in LTM (i.e., ALTM). Specifically, we measured indirect or semantically mediated priming during and after WM demands that focused attention on specific content. In addition, we investigated whether the type of attention-driven processing in WM would affect the magnitude of priming (e.g., rehearsal operations vs. semantically complex operations, such as category identification). Finally, we tested whether ALTM processes (as assessed with semantic priming) are limited by the same resources that constrain WM attention processes (i.e., do concurrent attentional WM demands affect the magnitude of ALTM?).

With most of the tasks used in previous WM research (e.g., WM span tasks), the temporary availability of LTM has not been assessed independently of attention-driven WM components. Those tasks that do distinguish between the two tend to focus primarily on the availability of justprocessed information that is no longer in the focus of attention (e.g., Haarmann et al., 2003; Oberauer, 2002). Consequently, a new experimental task was developed that combined a short-term verbal retention component resembling conventional WM demands (i.e., concurrent storage and processing) and a semantic priming component used to assess increased availability of information related to what has just been processed. The novel element of this experimental task was the use of these two components within a single processing task, so that ALTM and attention-driven WM processes could be investigated concurrently.

The ALTM task has four trial components, and the order of three of the components can be varied across experiments. Figure 1 presents a summary of these trial components with example trial content. The first component is always a memory load that consists of a set of four words (usually two syllables) presented one at a time for eventual recall. Within each memory load, some of the words (usually half) represent one semantic category, and the remaining words represent another category. In Figure 1, the two semantic categories are precious stones and relatives. We assume that the memory load phase of each trial engages participants' attention-driven WM processes for active rehearsal.

A second type of trial component, referred to as the selection instruction, introduces a concurrent demand that increases the amount of processing devoted to one of the two categories in the memory load. This is accomplished with an instruction to identify and remember the words from just one of the categories. This instruction could take several forms, including remember the relatives or ignore the precious stones. We assumed that this trial component engages attention-driven category identification processes for one category concurrently with ongoing memory load maintenance. Furthermore, after the target category exemplars are selected by the participant, we assume that subsequent rehearsal processes focus on only the target category exemplars. In total, more attentional resources are presumably devoted to one of the two memory load categories in the form of both category identification processes and rehearsal processes.

A third type of trial component consists of recall of the memory set elements that were supposed to be remembered. Performance on this component was not of primary interest but served to verify that individuals were adequately performing the conventional WM task demands represented in the first two task components.

The final type of trial component consists of samedifferent category membership comparisons that assess 


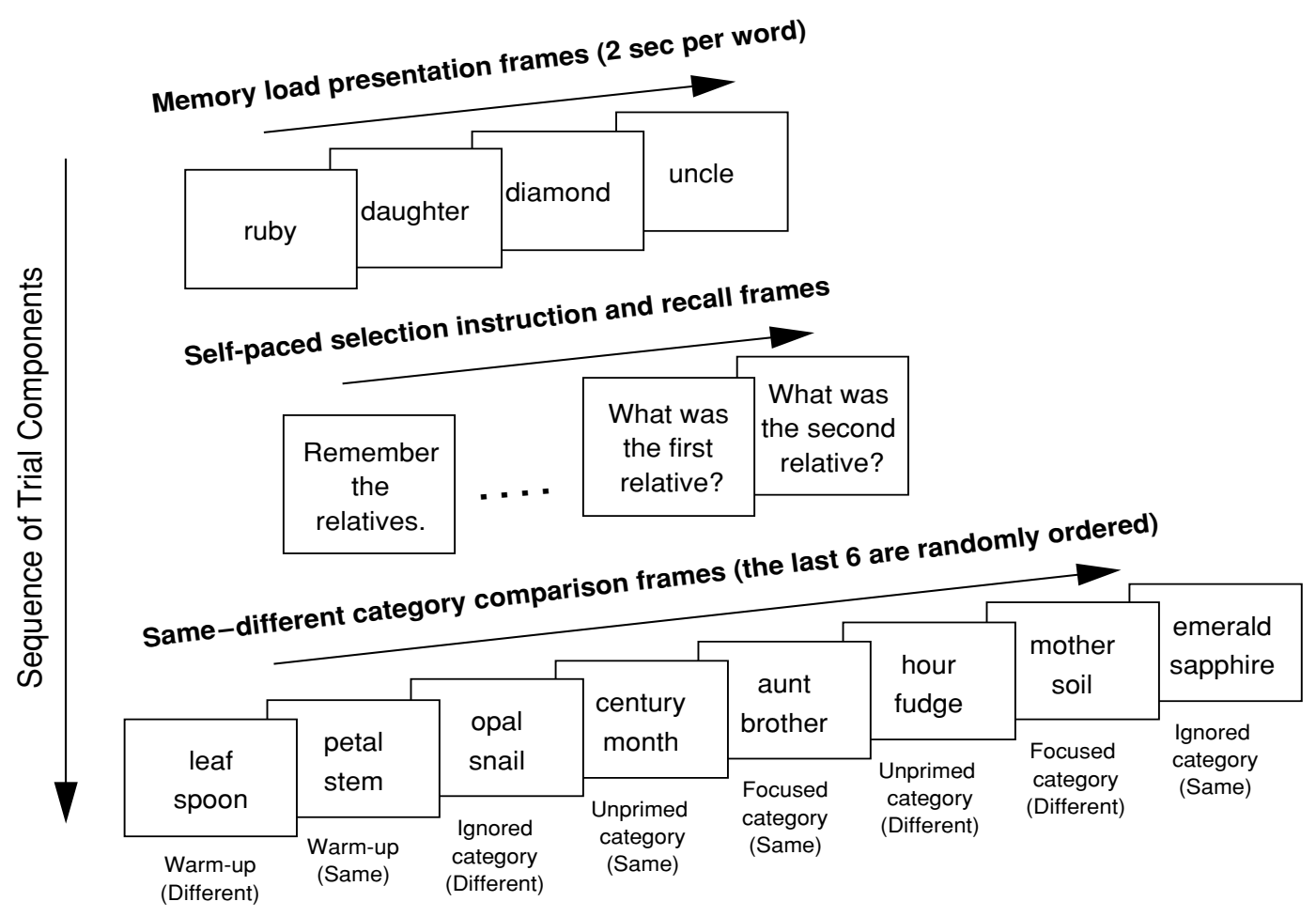

Figure 1. Example trial of the ALTM task used in Experiment 1.

priming or increased availability of the categories represented in the memory load. Each category comparison consists of two exemplars to which the participant responds like if they are exemplars from the same category (e.g., aunt brother) or different if they are exemplars from different categories (e.g., mother soil). Category comparisons in the present experiments never contained exemplars that were the same as those presented in the memory load. Within each trial, they contained new exemplars from three categories: the focused category from the memory load (i.e., the category that was identified for eventual recall), the ignored category from the memory load, and an unprimed category that was not represented in the memory load. We infer ALTM from increased response speed for comparisons representing the two memory load categories (focused and ignored categories), relative to the unprimed category. Because exemplars used in the category comparisons were never those rehearsed in the memory load, any facilitation observed in the focused and ignored category comparisons can be interpreted as increased availability of the general category representations in LTM, rather than as increased availability of the specific exemplars that were processed. Finally, contrasting response speed for focused and ignored category comparisons allows for the assessment of differences in ALTM as a function of different amounts and types of attention-driven processing in the prior memory load phase.

Four experiments were conducted using variations of this task. In Experiments 1 and 2, we examined the relationship between the content and processing demands of the memory load phase and the resulting availability of related information, as revealed in priming during the category comparison phase. In Experiments 3 and 4, we examined the relative impact of prior versus concurrent memory load maintenance and category selection on priming in the category comparison phase.

\section{EXPERIMENT 1}

The purpose of this experiment was to test the fundamental assumption that attention-driven processes in WM focused on specific verbal content produce an increased availability in conceptually related LTM representations. In this experiment, the category comparisons (i.e., the priming measure) always followed the memory load, selection instruction, and recall components. Consequently, the facilitation measure in this experiment assessed whether related information was more available subsequent to attention-based processing in WM. In addition, the comparison of priming in focused versus ignored categories from the memory load tested whether ALTM differed according to the amount of content-specific prior processing in WM.

\section{Method}

Participants. Forty-eight undergraduate students ( $75 \%$ female) participated in this experiment for credit in an undergraduate educational psychology course. Median age of the sample was 23 years (range, 19-49).

Apparatus. The participants performed the experimental task on IBM-compatible microcomputers with SVGA monitors and stan- 
dard keyboards. The experiment was programmed with E-Prime software (Schneider, Eschman, \& Zuccolotto, 2002).

Experimental task. As has been described earlier, the experimental task consisted of four components: memory load presentation, selection instruction, memory load recall, and category comparison frames. Each of 24 trials contained the four components in the described order. The stimuli used in the 24 trials are contained in the Appendix. The categories and exemplars were obtained primarily from category norms by Battig and Montague (1969), but some additional categories and exemplars were generated. We chose relatively frequent exemplars for each category, because we wanted there to be little or no ambiguity regarding category membership. However, no attempt was made to eliminate homographs from the stimuli, because we felt that such words represent everyday meaning retrieval demands.

As is shown in the Appendix, categories were organized in 24 sets, with each set containing three categories. The category triplets were chosen so that there was minimal conceptual overlap among the three categories. For each participant, one category from each set was assigned to be the focused category in the memory load, one was assigned to be the ignored category in the memory load, and the remaining one represented a category not found in the memory load. Six versions of the experiment were created that represented a complete counterbalancing of triplet category assignment to priming condition (focused, ignored, and unprimed).

Each trial began with the statement Get ready to memorize words displayed for $4 \mathrm{sec}$, followed by a blank screen for $1 \mathrm{sec}$. This was followed by four words presented on the display one at a time. Each word set was preceded by an asterisk displayed for $750 \mathrm{msec}$ in the location of the words (center of screen) and then a blank screen for $1 \mathrm{sec}$. Each word was displayed for $1,500 \mathrm{msec}$, followed by a blank screen for $500 \mathrm{msec}$. Order of the memory load words was constrained so that the exemplars from a category could not be contiguous (i.e., categories were alternated). Which category came first was randomized.

There was a 2-sec delay after the final memory load item. This was followed by an instruction frame that directed the participant to remember only two of the four terms in the memory load. The instruction always named the category to be remembered, rather than the category exemplars (e.g., Remember words that are fruit). The participants could take as long as needed to identify and rehearse the two target exemplars in the memory load. They were instructed to press the space bar when ready to proceed.

Following the selection instruction and a 1-sec delay, the participants were prompted to recall the two words held in memory. There were two recall frames that asked, What was the $<$ first, second $>$ word that was a $<$ category name $>$ ? The participants were instructed to type the first two letters of each word they were recalling.

Following the second recall frame, there was a 1-sec delay, followed by the instruction, Get ready to COMPARE words . . Rest your fingers on the $L$ and $D$ keys. This instruction was presented for $3 \mathrm{sec}$, followed by a 2-sec blank screen to allow the participants to prepare for the comparison frames. Each comparison frame began with two asterisks presented for $500 \mathrm{msec}$, one on top of the other at the location at which the two stimulus words would appear. The asterisks were followed by a blank screen for $750 \mathrm{msec}$ and then the two stimulus words. The stimuli remained on the screen until the participant responded by pressing either the L or the D key. A 1-sec interval separated the response and the asterisks preceding the subsequent comparison. During the entire set of comparison frames, the lower left portion of the display contained the reminder $D=$ Different, and the lower right portion of the display contained $L=L i k e$. As was described earlier, the participants were instructed to decide whether the two exemplars in each comparison came from the same category ( $\mathrm{L}$ response) or different categories (D response).

There was a total of eight category comparison frames in each trial. The first two frames were warm-ups that contained words unrelated to the contents of the memory load and the unprimed category in the stimulus set. The remaining six frames were in random order for each participant. They consisted of two frames each, one positive match and one negative match, for the three categories of the stimulus set (focused, ignored, and unprimed).

Following the category comparison frames, feedback was provided for the entire trial. The participants were informed of their accuracy for the recall frames and their average accuracy and response time for the category comparison frames. Prior to the next trial, the participants were reminded that they should try to obtain perfect accuracy on the recall frames and try to respond as quickly as possible without making errors on the category comparison frames. The feedback and goal reminder frames were self-paced.

Procedure. The participants performed the experimental task in a single 1-h session. They performed the experiment in groups of 1-6, with each participant seated in a computer carrel separated by sounddeadening panels. Equal numbers of participants performed the six counterbalanced versions of the experiment ( $n=8$ per version).

\section{Results}

The participants were relatively accurate in selecting and recalling the focused category words from the initial memory loads. Mean accuracy was greater for the first exemplar $(M=96.4 \%, S D=6.1 \%)$ than for the second exemplar $(M=92.2 \%, S D=7.2 \%)[F(1,47)=32.22$, $\left.M S_{\mathrm{e}}=12.92 ; \eta^{2}=0.41\right]$. These accuracy means were probably conservative estimates of performance on the memory load and recall phases, because we applied a strict scoring procedure that counted all responses other than the correct two letters as an error. In examining the recall errors, $30 \%$ of the errors appeared to be phonetically based misspellings (e.g., sa for sergeant, we for Wheaties, gi for guitar, pe for Purdue, ca for kerosene, etc.). Another $10 \%$ of the recall responses were counted as errors because they had either only one letter or more than two letters, even though they were the correct letter(s). One third of the errors could not be classified in any obvious way, but some recall errors did appear to reflect WM failures. Eight percent of the errors were blank responses. These were predominantly in the second term, perhaps reflecting the loss of the second term while responding to the first recall probe. Fifteen percent of the error responses reflected exemplars in the wrong serial order. It should be noted that no participant made more than one of these order errors, so these errors do not reflect misunderstanding of the importance of recall order in the task. Finally, $6 \%$ of the errors appeared to reflect incorrect selection of the focused category exemplars (i.e., they corresponded to correct responses for an exemplar from the ignored category). Overall, the participants were quite accurate in recalling the correct exemplars, and there was no predominant type of error in the incorrect responses.

The hypotheses about ALTM were tested with a dependent variable created by transforming and combining response time and response accuracy variables. Because priming effects were evident in both latency and accuracy measures, a combined measure was computed for each trial condition as the participant's number correct for comparisons in that condition divided by the sum of response latency for all the comparisons in that condition (both correct and incorrect). This measure represents an index of response speed adjusted for errors: It is the recip- 
rocal of response latency, and it adjusts speed according to errors. It can be interpreted directly as number of correct responses per unit time (minutes in this case). This index has the advantage of incorporating meaningful variance of both latency and error rates, and the distribution usually approximates the normal distribution more closely than does that of either the latency or the error distribution. All statistical tests corresponding to our hypotheses were conducted using this dependent variable. However, the mean latency and error data from which the speed scores were computed are reported in Table 1 (and subsequent tables for each experiment).

Figure 2 shows the average response speed by trial condition. Statistical analyses were conducted separately for positive and negative match comparisons for two reasons. First, hypotheses about priming effects primarily concerned positive match comparisons. Both theory and prior evidence suggest that priming should be minimal or nonexistent on negative match comparisons in which different categories are compared (e.g., Woltz, 1990). Second, the stimuli were not randomly assigned to positive and negative match conditions. So, any effect of match type found in a combined analysis could not be interpreted, due to the confounding of stimulus content and match type.

Hypothesized priming effects for the ignored and focused category stimuli were analyzed using a repeated measures ANOVA with two orthogonal contrasts. Alpha was set at .05. The first contrast compared the average for ignored and focused comparisons with that for unprimed comparisons, in order to test the overall priming effect of having category exemplars temporarily maintained and processed in WM. The second contrast compared ignored and focused category comparisons, in order to test whether the degree of attention devoted to category exemplars in WM affected the degree of residual ALTM.

As can be seen in Figure 2, for negative match comparisons, there were minimal differences in response speed among the three comparison types (focused, ignored, and unprimed). There was no overall priming effect $[F(1,47)<1]$, and the difference between ignored and focused category comparison speeds did not reach statistical significance either $\left[F(1,47)=3.99, M S_{\mathrm{e}}=21.67, p>.05\right]$.

Table 1

Mean Error Rates (in Percentages) and Response Latencies (in Milliseconds) for Category Comparisons by Match Type and Trial Condition in Experiment 1 (With Standard Deviations)

\begin{tabular}{lccccc}
\hline & \multicolumn{3}{c}{ Positive Match } & & \multicolumn{2}{c}{ Negative Match } \\
\cline { 2 - 3 } \cline { 5 - 6 } Comparison Type & $M$ & $S D$ & & $M$ & $S D$ \\
\hline & \multicolumn{3}{c}{ Error Rates } & & \\
Unprimed & 6.5 & 6.3 & & 4.6 & 6.0 \\
Ignored category & 5.0 & 5.1 & 4.6 & 6.0 \\
Focused category & 3.5 & 4.0 & 5.1 & 5.8 \\
& Response Latencies & & \\
Unprimed & 1,090 & 313 & 1,244 & 387 \\
Ignored category & 1,045 & 296 & 1,266 & 419 \\
Focused category & 1,030 & 304 & 1,220 & 383 \\
\hline
\end{tabular}

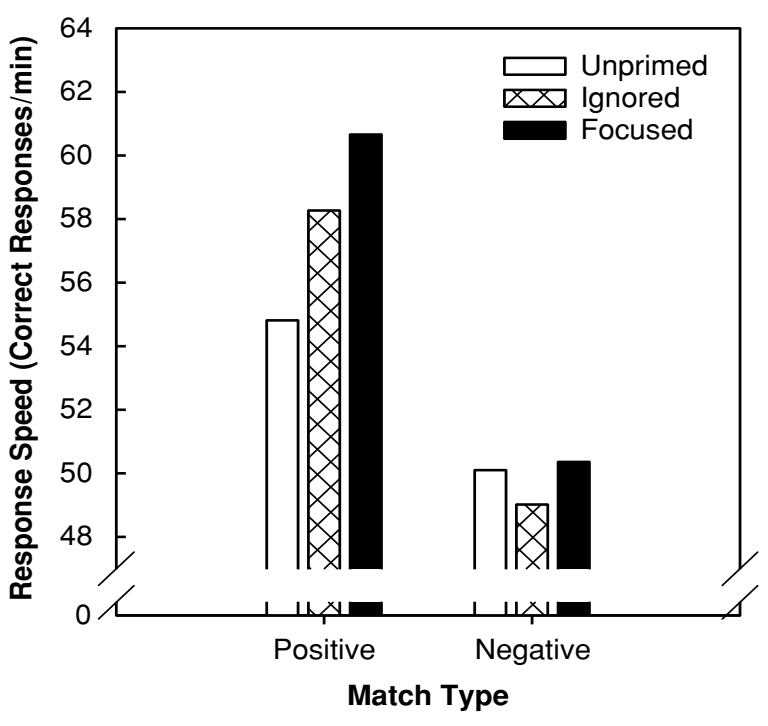

Figure 2. Mean response speed (correct responses per minute) for category comparisons in Experiment 1 by priming condition and match type.

For positive match comparisons, Figure 2 shows a notable difference in response speed among the three comparison types. The participants were faster in responding to ignored and focused category comparisons than to unprimed comparisons $\left[F(1,47)=21.07, M S_{\mathrm{e}}=\right.$ $\left.1,041.72 ; \eta^{2}=0.31\right]$. In addition, the participants were faster in responding to focused category comparisons than to ignored category comparisons $\left[F(1,47)=7.72, M S_{\mathrm{e}}=\right.$ 275.44; $\left.\eta^{2}=0.14\right]$.

Finally, we made an additional comparison between the ignored and the unprimed category comparisons, in order to test whether there was reliable priming for the ignored category (i.e., did minimal rehearsal and subsequent rejection of these category exemplars result in measurable facilitation?). The participants were faster in responding to ignored category comparisons than to unprimed category comparisons $\left[F(1,47)=9.67, M S_{\mathrm{e}}=287.46 ; \eta^{2}=\right.$ $0.17]$.

\section{Discussion}

These results demonstrate how attention-driven processing of category exemplars in WM resulted in greater availability of related category information in LTM and how the amount of processing in WM affected the degree of this subsequent availability. We attribute the priming effects in the category comparisons entirely to the previous memory load processing, despite the fact that there could be some level of priming among semantically related frames in the comparison phase of each trial. Recall that there was one negative and one positive match comparison for each category within a trial, randomly ordered for each participant. However, we do not believe that this could account for the observed priming effects. First, prior evidence suggests that semantically related positive and 
negative match comparisons produce little or no priming for one another (Woltz, 1990). Second and more important, if there was between-comparison priming, it would exist in the comparisons for the unprimed category, as well as for the two primed categories. Priming was evaluated as the difference between the unprimed category and the focused and ignored categories.

These results have importance for new conceptualizations of WM for several reasons. First, the priming effects observed were specific to the semantic categories processed in working memory, rather than to the exemplars processed. This corresponds to one of the underlying rationales for Ericsson and Kintsch's (1995) and Kintsch et al.'s (1999) proposals of long-term WM: Normal comprehension ability among readers requires the availability of far more information than can be contained in traditional attention-driven models of WM. Furthermore, much of the information that must be available in WM is prior knowledge that is associated with, but not directly contained in, the text that is processed.

Second, in addition to being category specific, rather than exemplar specific, the priming effects were relatively long-lasting. The six category comparisons corresponding to the unprimed, ignored, and focused categories always followed two warm-up comparisons. So, on average, the positive match primed comparison for each category occurred five comparison frames after the memory load recall. The presence of priming after this amount of intervening processing again corresponds to the rationale behind some of the new conceptualizations of WM. To explain human comprehension abilities, it is necessary to assume that a relatively large amount of semantic information is available for processing for more than a few seconds.

Third, the priming effects observed in this experiment were affected by the degree of processing in WM. This is important because it demonstrates a direct relationship between controlled attentional processes of WM and the presumably automatic activation or retrieval processes in LTM. Furthermore, the results demonstrated increased availability for semantic content that had relatively little attention devoted to it. The exemplars representing the ignored category were initially encoded and rehearsed along with the focused category exemplars. However, almost immediately, the participants were instructed to remember only the focused category exemplars. So, rehearsal time was minimal for the ignored category exemplars, and their category name was never presented. Yet this minimal processing produced reliable facilitation in semantically related exemplars. This suggests that the effective capacity of ALTM resulting from WM temporary maintenance processing is probably extensive.

Although the experiment provided a general demonstration of how attentional processing in WM results in ALTM, one particular outcome of the experiment has several possible interpretations. The difference between ignored and focused priming could be due to (1) more time of the focused category exemplars in an attention-driven rehearsal loop, (2) explicit processing of the focused category name during the selection instructions and the ensuing identification of the exemplars as category members, or (3) both of these. The data from the present experiment cannot address these different interpretations, so Experiment 2 was designed for that purpose.

\section{EXPERIMENT 2}

The purpose of this experiment was to assess the relative contributions of rehearsal and category identification in the priming of related LTM content. This was done by varying whether the participants were instructed to select exemplars of a particular category (i.e., the procedure in Experiment 1) or to reject exemplars from another category (i.e., reject exemplars from a named category and remember the others by default, without naming the category to be remembered). If attention processes of rehearsal were solely responsible for the pattern of priming effects in Experiment 1, there should be no difference between trials containing select versus reject instructions in this experiment. If priming differences were due entirely to category naming during the selection instruction, priming of ignored category exemplars should be as great following a reject instruction as priming of the focused category exemplars following a select instruction, and there should be no priming for categories that were not explicitly identified in the instruction.

\section{Method}

Participants. Seventy-two undergraduate students (74\% of them female) participated in this experiment for credit in an undergraduate educational psychology course. Median age of the sample was 23 years (range, 19-51).

Apparatus. The participants performed the experimental task on IBM-compatible microcomputers with SVGA monitors and standard keyboards. The experiment was programmed with E-Prime software (Schneider et al., 2002).

Experimental task. The experimental task was identical to that in Experiment 1, with the following modifications. First, half of the trials for each participant contained a reject instruction, rather than a select instruction. The select instruction was identical to that in Experiment 1 (e.g., Remember words that are relatives). The reject instruction was worded, Ignore words that are precious stones. Trial content (see the Appendix) was randomly assigned to reject and select instruction conditions for each participant. Second, the recall instruction frames no longer labeled the focused category, as in Experiment 1. The recall instructions were now worded, What was the $<$ first, second $>$ word that you were supposed to remember? Thus, trials with the select instruction were comparable to those in Experiment 1, except that the category was not named during the recall probes. Trials with the ignore instruction still resulted in a focused category whose exemplars received additional rehearsal and recall. However, the focused category name was never presented to the participants (either during the memory load instruction or during recall). Instead, during the reject trials, the ignored category name was presented during the memory load phase, and the ignored category exemplars had to be identified and rejected.

Procedure. The participants performed the experimental task in a single 1-h session. They performed the experiment in groups of $1-6$, with each participant seated in a computer carrel separated by sound-deadening panels. 


\section{Results}

Only data from positive match comparisons were analyzed, given the results of Experiment 1 and the lack of theoretical motivation for investigating priming effects in negative match comparisons. Table 2 presents the mean latency and error data for positive match category comparisons by trial condition. Figure 3 presents the mean adjusted response speed data for positive match category comparisons by trial condition.

The reject instruction trials appeared to be slightly more difficult during the memory load and recall phases. For select instruction trials, the mean time taken for selecting the identified exemplars was 4,231 msec $(S D=$ $1,286)$, and the mean recall accuracy for the first and second terms, respectively, was $M=96.8 \%(S D=5.3 \%)$ and $M=94.3 \%(S D=8.5 \%)$. For reject instruction trials, the mean time taken for selecting the identified exemplars was $4,947 \mathrm{msec}(S D=1,615)$, and the mean recall accuracy for the first and second terms was $M=92.4 \%(S D=$ $9.2 \%)$ and $M=89.4 \%(S D=10.6 \%)$, respectively. The effect of instruction type on selection time was statistically significant $\left[F(1,71)=44.66, M S_{\mathrm{e}}=413,422 ; \eta^{2}=\right.$ $0.39]$, as was the effect on recall accuracy $[F(1,71)=$ $\left.17.12, M S_{\mathrm{e}}=92.41 ; \eta^{2}=0.19\right]$. As in Experiment 1, there was also an effect of order on recall accuracy $[F(1,71)=$ $\left.19.74, M S_{\mathrm{e}}=26.98 ; \eta^{2}=0.22\right]$, but there was no interaction of order and instruction type $[F(1,71)<1]$.

As is shown in Figure 3, there was an overall priming effect similar to that found in Experiment 1. The contrast of ignored and focused category comparisons with unprimed category comparisons was statistically significant $[F(1,71)=$ $\left.36.65, M S_{\mathrm{e}}=1,943.37 ; \eta^{2}=0.34\right]$. However, the contrast of focused versus ignored category comparisons was not $[F(1,71)<1]$. Instead, there was a disordinal interaction between primed comparison type (focused vs. ignored) and memory load instruction $\left[F(1,71)=4.68, M S_{\mathrm{e}}=407.02\right.$; $\left.\eta^{2}=0.06\right]$. Mean response speed was greater for focused categories when there was a select instruction during the memory load phase and was greater for the ignored categories when there was a reject instruction.

Additional comparisons were conducted to examine two issues. First, we analyzed whether response speed differed

Table 2

Mean Error Rates (in Percentages) and Response Latencies (in Milliseconds) for Positive Match Category Comparisons by Condition in Experiment 2 (With Standard Deviations)

\begin{tabular}{lccccc}
\hline & \multicolumn{2}{c}{$\begin{array}{c}\text { Select } \\
\text { Instruction }\end{array}$} & & \multicolumn{2}{c}{$\begin{array}{c}\text { Reject } \\
\text { Instruction }\end{array}$} \\
\cline { 2 - 3 } \cline { 5 - 6 } Comparison Type & $M$ & $S D$ & & $M$ & $S D$ \\
\hline & \multicolumn{2}{c}{ Error Rates } & & \\
Unprimed & 7.1 & 7.6 & & 7.4 & 7.9 \\
Ignored category & 5.0 & 5.5 & & 2.6 & 5.0 \\
Focused category & 4.5 & 6.7 & & 3.6 & 5.4 \\
& Response Latencies & & \\
Unprimed & 1,061 & 244 & & 1,089 & 284 \\
Ignored category & 1,058 & 258 & & 1,036 & 236 \\
Focused category & 1,021 & 222 & & 1,062 & 295 \\
\hline
\end{tabular}

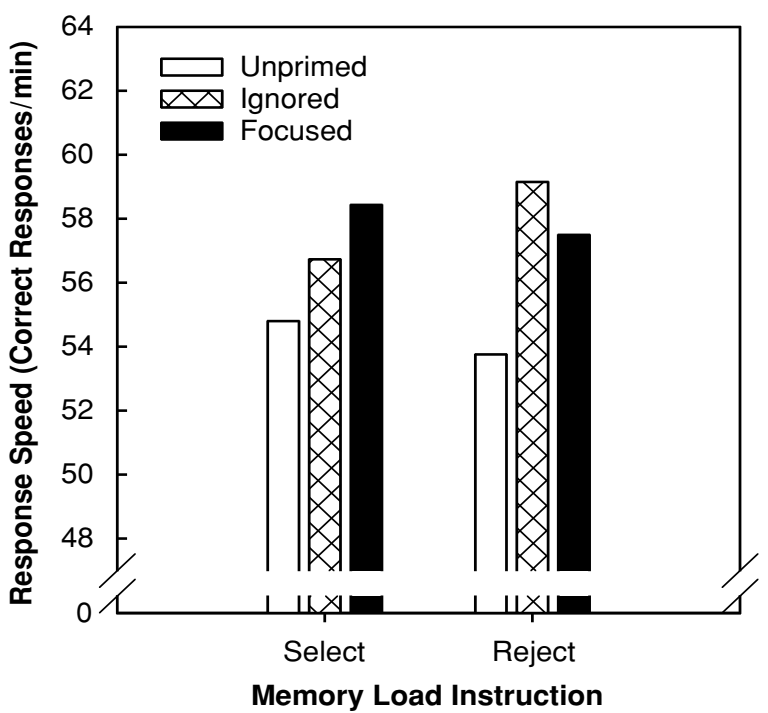

Figure 3. Mean response speed (correct responses per minute) for positive match category comparisons in Experiment 2 by priming condition and memory load instruction.

between ignored category comparisons following reject instructions and focused category comparisons following select instructions. The difference in response speed between these two conditions of maximal priming was not statistically significant $[F(1,72)<1]$. This suggests that attention-driven semantic processing of the content of WM was more instrumental in producing ALTM than was the rehearsal processing. If rehearsal was of additional importance in producing ALTM effects, priming in the focused categories should have been greater, because of the increased rehearsal time for these category exemplars.

A second comparison tested whether there was measurable priming for categories that were not explicitly identified in the memory load instruction. In other words, was category naming during the memory load instruction necessary for priming to occur? For ignored category comparisons following select instructions, the priming effect (i.e., the contrast of these comparisons with unprimed category comparisons) approached but did not reach statistical significance $[F(1,71)=3.33, p=.07]$. However, for focused category comparisons following reject instructions, there was statistically significant priming $\left[F(1,71)=11.17, M S_{\mathrm{e}}=502.79 ; \eta^{2}=0.14\right]$. These results suggest that some priming occurs in the absence of category naming in the memory load instruction, particularly when category exemplars are actively maintained in WM. Although the ignored category priming did not reach statistical significance, the same condition in Experiment 1 , which had twice the number of trials per condition, did produce reliable priming.

\section{Discussion}

The results of this experiment are consistent with the view that ALTM is determined more by the conceptually driven operations performed on what is actively main- 
tained in WM (i.e., category membership identification) than by the rehearsal processes themselves. The disordinal interaction between category comparison content and memory load instruction provided direct support for this interpretation. However, as in Experiment 1, even minimal processing and rehearsal in WM produced some measurable priming. The focused category trials showed priming even during the reject instruction condition. Thus, semantic labeling and selection processes have the greatest impact on ALTM, but even simple encoding and rehearsal processes appear to have some impact.

\section{EXPERIMENT 3}

In the previous experiments, we investigated ALTM following WM task demands with delays of approximately 5-30 sec. In the present experiment, we investigated the patterns of ALTM during, rather than after, maintenance of a WM load. The primary theoretical issue of interest was whether attention-driven maintenance processes of WM and processes underlying ALTM draw upon the same cognitive resources. If so, one would expect priming to be attenuated with the concurrent demand of maintaining a memory load. This potential reduction in priming might be offset by renewed source activation from maintenance processing (i.e., rehearsal). However, this could occur only with semantic content related to the concurrent memory load (i.e., in the focused category and not in the ignored category). Furthermore, the results from Experiment 2 suggest that the nature of semantic processing is more instrumental to priming than is maintenance processing, so the increased source activation during rehearsal would not be expected to be a major factor. In sum, if ALTM processes that emanate from attention processes represent a distinct set of cognitive limits in cognition, one would expect priming with and without a concurrent memory load to be equivalent for content unrelated to the memory load, and one might even expect this for content related to the memory load.

\section{Method}

Participants. Ninety undergraduate students $(73 \%$ of them female) participated in this experiment for credit in an undergraduate educational psychology course. Median age of the sample was 25 years (range, $18-52$ ).

Apparatus. The participants performed the experimental task on IBM-compatible microcomputers with SVGA monitors and standard keyboards. The experiment was programmed with E-Prime software (Schneider et al., 2002).

Experimental task. The experimental task was identical to that used in Experiment 1, except that the recall probes occurred after, rather than before, the category comparisons for a random half of the 24 trials, thus producing a concurrent memory load of the focused category exemplars during the comparison frames. In addition, recall instructions were worded as in Experiment 2, with no explicit labeling of the category to be recalled.

Procedure. The participants performed the experimental task in a single 1-h session. They performed the experiment in groups of $1-6$, with each participant seated in a computer carrel separated by sound-deadening panels.

\section{Results}

As in Experiment 2, only data from positive match comparisons were analyzed. Table 3 presents the mean latency and error data by category comparison condition for positive match trials. Figure 4 presents the means of category comparison response speed by trial condition.

When recall occurred prior to the category comparisons, mean recall accuracy for the first and second terms was $M=96.9 \%(S D=6.3 \%)$ and $M=93.8 \%(S D=$ $9.0 \%$ ), respectively. When recall followed the category comparisons, mean recall accuracy for the first and second terms was $M=96.7 \%(S D=6.6 \%)$ and $M=93.7 \%$ $(S D=8.5 \%)$, respectively. A $2 \times 2$ ANOVA was conducted, with memory load term (first vs. second) and recall order (before vs. after the category comparisons) as within-subjects independent variables. The effect of memory load term was statistically significant $[F(1,89)=$ $\left.34.22, M S_{\mathrm{e}}=814.90 ; \eta^{2}=0.28\right]$. However, the effect of recall order was not $[F(1,89)<1]$. Thus, the participants were able to effectively maintain the memory load during the category comparisons.

As can be seen in Figure 4, there were overall priming effects similar to those in the previous two experiments. The contrast of ignored and focused category comparisons with unprimed category comparisons was statistically significant $\left[F(1,89)=34.92, M S_{\mathrm{e}}=1,649.84 ; \eta^{2}=\right.$ 0.28]. As in Experiment 1, the difference between ignored and focused category comparisons was also significant $\left[F(1,89)=6.46, M S_{\mathrm{e}}=503.94 ; \eta^{2}=0.07\right]$. Of primary interest in this experiment was whether the magnitude of these two priming effects (overall priming and focusspecific priming) would differ when the memory load maintenance operations were performed prior to, versus concurrent with, the category comparisons. As can be seen in Figure 4, there was a reduction in overall response speed for all the comparison conditions when the memory load was concurrent $\left[F(1,89)=12.89, M S_{\mathrm{e}}=\right.$ $\left.284.71 ; \eta^{2}=0.13\right]$. However, there was no evidence for an interactive effect of memory load maintenance on either priming contrast $[F(1,89)<1$ for both].

Table 3

Mean Error Rates (in Percentages) and Response Latencies (in Milliseconds) for Positive Match Category Comparisons by Condition in Experiment 3 (With Standard Deviations)

\begin{tabular}{lccccc}
\hline & \multicolumn{2}{c}{$\begin{array}{c}\text { Prior Memory } \\
\text { Load }\end{array}$} & & \multicolumn{2}{c}{$\begin{array}{c}\text { Concurrent } \\
\text { Memory Load }\end{array}$} \\
\cline { 2 - 3 } \cline { 5 - 6 } Comparison Type & $M$ & $S D$ & & $M$ & $S D$ \\
\hline & \multicolumn{2}{c}{ Error Rates } & & \\
Unprimed & 6.0 & 7.3 & & 6.0 & 6.3 \\
Ignored category & 4.1 & 5.8 & & 5.4 & 6.5 \\
Focused category & 3.6 & 5.6 & & 3.7 & 6.7 \\
& Response Latencies & & \\
Unprimed & 1,154 & 434 & & 1,199 & 397 \\
Ignored category & 1,114 & 365 & & 1,171 & 396 \\
Focused category & 1,091 & 356 & 1,156 & 392 \\
\hline
\end{tabular}




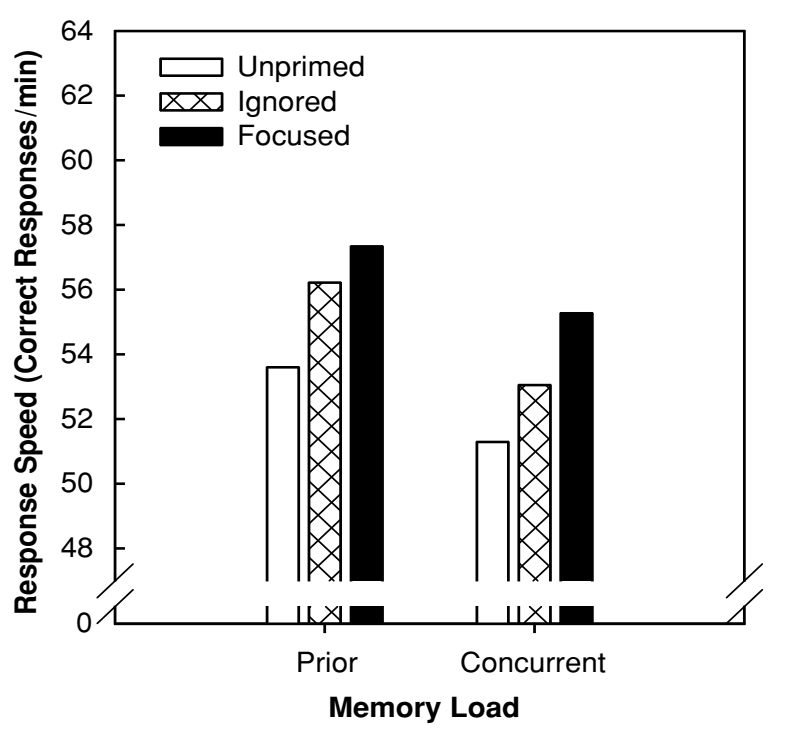

Figure 4. Mean response speed (correct responses per minute) for positive match category comparisons in Experiment 3 by priming condition and memory load condition.

Although interactions of memory load maintenance and both contrasts of comparison type were not statistically significant, there was a trend for priming in the ignored category to be reduced during the concurrent load, in comparison with the prior load (see Figure 4). This would be consistent with a view that maintenance processing of the focused category exemplars during the concurrent load condition reduces the availability of other LTM structures. We explored this trend by comparing only the ignored and the unprimed category comparisons by recall order. As in Experiment 1, there was a significant priming effect in the ignored category comparisons $[F(1,89)=13.65$, $\left.M S_{\mathrm{e}}=864.00 ; \eta^{2}=0.13\right]$. However, the interaction with memory load condition was not statistically significant $[F(1,89)<1]$. Again, we found no evidence that concurrent attention demands reduced ALTM, even for semantic content unrelated to the focused category.

\section{Discussion}

The results of this experiment suggest that the increased availability of LTM content that is semantically related to prior processing content is equivalent whether or not there is a concurrent memory load. This appeared to be the case when the semantic content of the category comparison was the focus of attention during selection and rehearsal of the memory load (focused category) and when it was not (ignored category). These findings are inconsistent with the view that ALTM and attention components of $\mathrm{WM}$ are dependent on a single limited resource. Instead, the magnitude of the ALTM appeared to be unaffected by the level and content of concurrent attention-driven maintenance processing. These findings are more consistent with a view that ALTM and attention processes in WM represent independent cognitive resources, or that pro- cesses underlying ALTM require no additional resources beyond the attention resources that initiated them.

Such a conclusion is open to several criticisms. First, it is based on a null finding, and the power to detect an effect is an issue. We increased the sample size in this experiment, relative to the previous experiments, in an attempt to address the power issue. However, the observed interactive effect of recall order was so small, relative to error variance, that the power of the statistical tests was still low $(<.10$ for both contrasts). We concluded that any potential effect that could be detected with a larger sample size would be so small as to be theoretically insignificant.

A second possible criticism is that priming is difficult to compare between prior and concurrent memory load conditions, because the baseline response speeds differed (i.e., the speed of unprimed comparisons was lower during the concurrent memory load). The question of concern is whether a 4-response/min facilitation effect reflects the same level of priming when the baseline rate is approximately 53 versus 51 responses $/ \mathrm{min}$. This is a scaling issue that we cannot resolve entirely. However, we assumed that the concurrent memory load did not increase baseline speed due to a qualitative change in category comparison processing. Rather, we assumed that the slowing reflected the need for the participants to periodically suspend attention to the comparison frames in order to rehearse the memory load. If this is the cause of slowing, we reasoned that the priming effects could be compared, because this additional processing, and corresponding slowing, should be equivalent in all comparison conditions in the concurrent load trials (i.e., unprimed, focused, and ignored comparisons).

A third possible criticism is that a concurrent memory load of two words did not sufficiently tax cognitive resources so as to impact ALTM processes. This possibility was addressed in the next experiment.

\section{EXPERIMENT 4}

The purpose of this experiment was to further test the question of independent versus shared resources for attention processes in WM and corresponding ALTM processes. This experiment was similar to Experiment 3, with the manipulation of concurrent versus prior memory load. However, the memory load was varied between one and three words, rather than always being two words. This tested the hypothesis that ALTM is reduced only when concurrent attention demands sufficiently tax capacity limits. That is, if ALTM and attention processes share a limited resource, ALTM should be diminished when concurrent attention demands are substantial (i.e., memory load of three 2-syllable words), in comparison with when they are minimal (i.e., memory load of one 2-syllable word).

\section{Method}

Participants. Seventy-eight undergraduate students (77\% of them female) participated in this experiment for credit in an undergraduate educational psychology course. Median age of the sample was 23 years (range, 18-51). 
Apparatus. The participants performed the experimental task on IBM-compatible microcomputers with SVGA monitors and standard keyboards. The experiment was programmed with E-Prime software (Schneider et al., 2002).

Experimental task. The task was identical to that used in Experiment 3 , except that for each participant, the 24 trials were randomly assigned to four conditions created by crossing the concurrent versus prior memory load manipulation and the manipulation of one versus three words in the focused category. Each trial still presented four words as the initial memory load, followed by an instruction to select a subset defined by category membership. However, three of the four words in the initial list were exemplars from one category, and the fourth word was from a different category (e.g., sofa apple table dresser). In half the trials, the select category instruction referred to the three-exemplar category (i.e., furniture), and in the other half, it referred to the one-exemplar category (e.g., fruit). Thus, the memory load maintenance demands following focused category selection varied between one and three words.

Procedure. The participants performed the experimental task in a single 1-h session. They performed the experiment in groups of $1-6$, with each participant seated in a computer carrel separated by sound-deadening panels.

\section{Results}

Figure 5 shows the mean recall data by memory load condition and recall term. In analyzing the recall data, we examined two sets of planned contrasts. First, we compared recall accuracy on the first term of the memory load by memory load size (one vs. three terms) and memory load maintenance (prior to vs. after category comparisons). There was no reliable effect of either maintenance or load on recall accuracy for the first term of a memory load ( $p \geq .20$ ). Thus, the primacy effect was not affected by the memory load conditions.

The second set of contrasts pertained only to trials with a memory load of three. As is evident in Figure 5, recall accuracy in these trials declined as a function of the serial order (linear) of the terms $\left[F(1,77)=21.66, M S_{\mathrm{e}}=0.34\right.$; $\left.\eta^{2}=0.22\right]$. Of primary importance, there was a statisti-

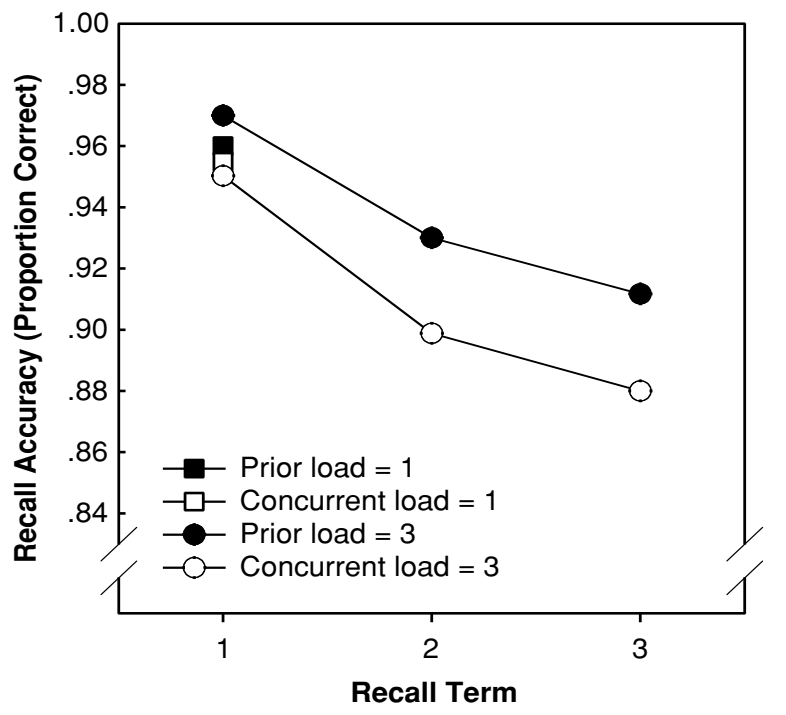

Figure 5. Mean recall accuracy (proportion correct) by trial condition in Experiment 4. cally significant effect of the memory load maintenance manipulation $\left[F(1,77)=4.11, M S_{\mathrm{e}}=0.10 ; \eta^{2}=0.05\right]$, with greater accuracy when the memory load was prior to the comparisons. This reflects the impact of concurrent processing demands (category comparisons) on memory load maintenance and recall. These data suggest that a concurrent memory of three 2-syllable words taxed WM resources. This was in contrast to Experiment 3, where recall accuracy was not affected by a concurrent memory load of two words.

As in the previous two experiments, only the data from positive match category comparisons are reported. Tables $4 \mathrm{~A}$ and 4B present the mean error and latency data, respectively, by trial condition. Figure 6 presents mean response speed for the category comparisons by condition.

Consistent with the results from the previous three experiments, there was an overall priming effect for categories represented in the memory load (focused and ignored) versus those not in the memory load (unprimed) $\left[F(1,77)=25.24, M S_{\mathrm{e}}=499.26 ; \eta^{2}=0.25\right]$. However, unlike in two of the previous experiments, the main effect for focused versus ignored category response speed did not reach statistical significance $[F(1,77)=2.63, p=.11]$. In the memory load 1 condition (left half of Figure 6), the participants responded correctly to ignored category exemplars with greater speed than they did to focused category exemplars, whereas the reverse was true in the memory load 3 condition (right half of Figure 6). This interaction of memory load and category (focused vs. ignored) was statistically significant $\left[F(1,77)=9.15, M S_{\mathrm{e}}=1,433.42\right.$; $\left.\eta^{2}=0.11\right]$ and presumably reflects the impact of number of exemplars from each category that were initially encoded. In memory load 1 trials, the initial memory load prior to focus category selection contained three ignored category exemplars and one focused category exemplar. In memory load 3 trials, the initial memory load contained three focused category exemplars and one ignored category exemplar. Consistent with findings from the earlier experiments, this interaction suggests that the semantic content of words encoded and rehearsed for even a brief time prior to focus category labeling and selection has a measurable impact on the availability of related LTM structures.

Another effect of importance was the interaction of memory load size (1 vs. 3 ) and memory load maintenance (prior vs. concurrent) $\left[F(1,77)=4.66, M S_{\mathrm{e}}=138.81\right.$; $\left.\eta^{2}=0.06\right]$. As can be seen in Figure 6, when the memory load was one word, the impact of concurrent versus prior load on response speed was negligible. In contrast, when the memory load was three words, there was a notable negative impact on response speed. This interaction is consistent with the assumption that maintaining a memory load of one word does not slow concurrent processes but that maintaining three words taxes attentional resources, so that concurrent processes are slowed.

Of primary importance was whether priming effects were reduced as a function of the increased concurrent memory load. There was no reliable effect on priming due to the memory load timing manipulation as tested by the interactions of prior versus concurrent load and category 
Table 4A

Mean Error Rates (in Percentages; With Standard Deviations) for Positive Match Category Comparisons by Condition in Experiment 4

\begin{tabular}{|c|c|c|c|c|}
\hline \multirow[b]{2}{*}{ Comparison Type } & \multicolumn{2}{|c|}{$\begin{array}{c}\text { Memory } \\
\text { Load = } 1\end{array}$} & \multicolumn{2}{|c|}{$\begin{array}{c}\text { Memory } \\
\text { Load }=3\end{array}$} \\
\hline & $M$ & $S D$ & $M$ & $S D$ \\
\hline \multicolumn{5}{|c|}{ Prior Memory Load } \\
\hline Unprimed & 8.8 & 13.6 & 10.1 & 12.1 \\
\hline Ignored category & 5.2 & 8.7 & 8.6 & 11.7 \\
\hline Focused category & 5.6 & 10.0 & 4.1 & 7.8 \\
\hline \multicolumn{5}{|c|}{ Concurrent Memory Load } \\
\hline Unprimed & 8.6 & 12.3 & 10.6 & 13.8 \\
\hline Ignored category & 4.7 & 9.7 & 7.8 & 10.3 \\
\hline Focused category & 4.3 & 8.7 & 3.9 & 9.0 \\
\hline
\end{tabular}

$[F(1,77)<1$ for both category contrasts]. Furthermore, priming was not reduced as a function of increased concurrent memory load as tested by the three-way interaction of memory load, recall order, and category $[F(1,77)<1$ for both category contrasts].

Finally, as in the previous experiments, it was of interest whether ignored category priming was reliable, even when minimal processing was devoted to that category. In the memory load 3 condition of this experiment, the ignored category had only one exemplar in the initial memory load, and that exemplar was rejected without an explicit label of the category following the memory load selection instruction. Despite this minimal processing, the participants responded correctly with greater speed to the ignored category comparisons than to the unprimed category comparisons in this memory load condition $\left[F(1,77)=4.49, M S_{\mathrm{e}}=93.94 ; \eta^{2}=0.06\right]$.

\section{Discussion}

As in Experiment 3, we found no evidence supporting the notion that increased concurrent attention demands reduce ALTM. When concurrent memory load maintenance demands increased, so that attention processes in WM appeared to be taxed, this had no measurable effect on priming in LTM. This was true for content related to the concurrent memory load and for content unrelated to

Table 4B

Mean Response Latencies (in Milliseconds; With Standard Deviations) for Positive Match Category Comparisons by Condition in Experiment 4

\begin{tabular}{|c|c|c|c|c|}
\hline \multirow[b]{2}{*}{ Comparison Type } & \multicolumn{2}{|c|}{$\begin{array}{l}\text { Memory } \\
\text { Load = } 1\end{array}$} & \multicolumn{2}{|c|}{$\begin{array}{l}\text { Memory } \\
\text { Load = } 3\end{array}$} \\
\hline & $M$ & $S D$ & $M$ & $S D$ \\
\hline \multicolumn{5}{|c|}{ Prior Memory Load } \\
\hline Unprimed & 1,158 & 378 & 1,098 & 323 \\
\hline Ignored category & 1,109 & 353 & 1,081 & 353 \\
\hline Focused category & 1,102 & 339 & 1,067 & 380 \\
\hline \multicolumn{5}{|c|}{ Concurrent Memory Load } \\
\hline Unprimed & 1,134 & 453 & 1,130 & 303 \\
\hline Ignored category & 1,088 & 310 & 1,137 & 349 \\
\hline Focused category & 1,148 & 394 & 1,106 & 305 \\
\hline
\end{tabular}

the concurrent memory load. As in the previous experiment, this evidence is consistent with a view that different cognitive resources underlie attention processes in WM and any additional processes underlying ALTM.

One could question whether a concurrent memory load of three semantically related words was sufficient to tax attentional resources. Although some earlier evidence suggests that a memory load consisting of three items does not always impact concurrent processes (Baddeley, 1986), the stimulus words used in this experiment averaged two syllables within each trial. Both anecdotal reports from the participants regarding the difficulty of the task and the recall accuracy data suggest that this memory load condition placed considerable demand on attentional resources. Furthermore, there was direct evidence that the concurrent memory load of three category exemplars slowed processing, in comparison with a load of one exemplar, in all comparison types (including unprimed category comparisons). Thus, in two experiments that varied the concurrent storage demands between one, two, and three 2 -syllable words, we found no evidence that temporary storage demands reduced priming in LTM for semantic categories either related to or unrelated to the concurrent memory load.

\section{GENERAL DISCUSSION}

Evidence from four experiments demonstrated a close linkage between demands for attention-driven processing within a WM task and the concurrent and subsequent priming of semantically related elements in LTM. This linkage was demonstrated with respect to two categories of attention-driven WM processes: temporary maintenance of memory load items and category membership identification of elements being maintained. With respect to the effect of memory load maintenance, Experiments 1 and 4 showed reliable priming for categories whose exemplars were not explicitly labeled or identified as the focus of further processing but were initially rehearsed for possible recall. The impact of the additional semantic processing of category membership identification was demonstrated in all the experiments. Identifying category membership of temporarily held exemplars increased priming above and beyond priming produced by simple maintenance processing. In particular, Experiment 2 showed that instructions to reject one category in order to focus on another category produced as much priming in the rejected category (i.e., the category requiring category membership identification) as in the subsequently retained and recalled category that did not explicitly require category membership identification. Also, Experiment 4 demonstrated that the magnitude of priming in both the focused and the ignored categories depended on the number of exemplars from these categories that were processed. In total, these findings suggest that the pattern of accessibility of LTM as measured by priming is closely tied to the amount and type of attention-driven processing in WM.

A link between the content of prior processing and patterns of subsequent processing facilitation is by no means 


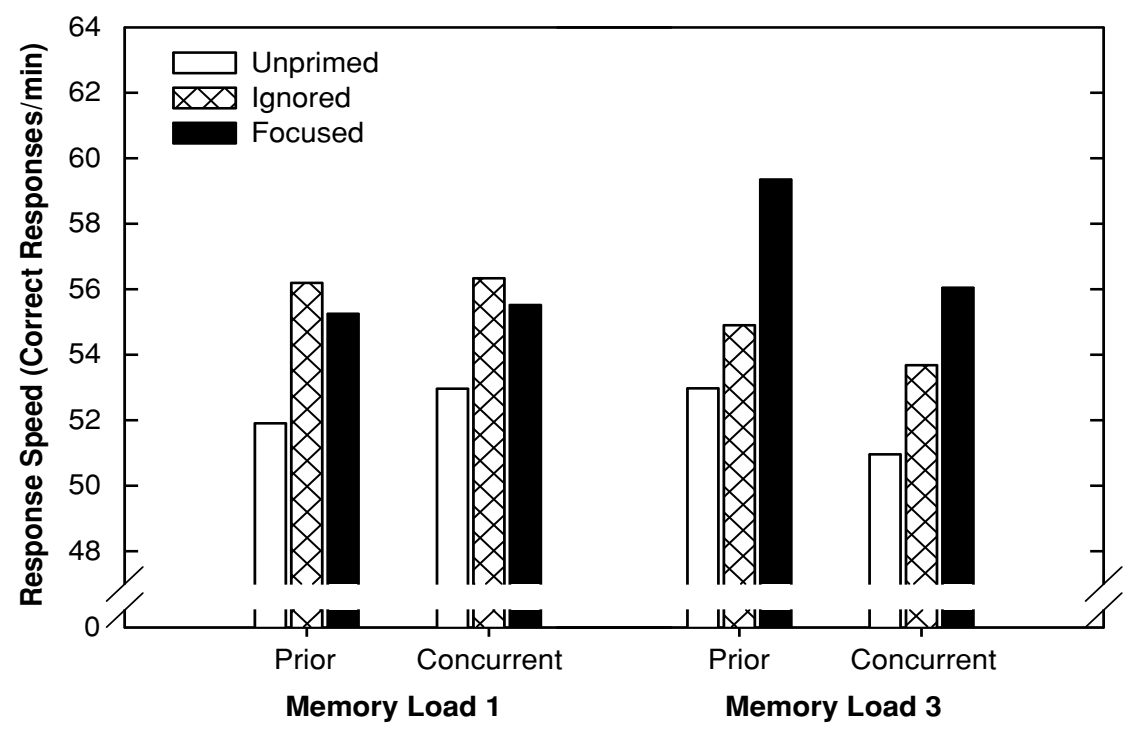

Figure 6. Mean response speed (correct responses per minute) for positive match category comparisons in Experiment 4 by priming condition, recall position, and memory load.

novel. The novelty of the linkages demonstrated here is that the priming was indirect or semantically mediated, rather than direct or repetition based. Second, the semantically mediated priming effects were relatively long-lasting, in comparison with most previously demonstrated semantic priming effects. As such, these results correspond to the notion that knowledge related to what has been in the focus of attention has an increased availability for subsequent processing and that this availability is somewhat persistent. Such persisting availability of related but unattended information has been suggested as a necessary component of complex comprehension processes (see, e.g., Ericsson \& Kintsch, 1995; Just \& Carpenter, 1992; Kintsch et al., 1999), but direct evidence for it in the context of WM models has been lacking.

\section{Magnitude and Persistence of the Priming Effects}

As has been noted, the general category priming effects demonstrated here were relatively long-lasting. Although semantic priming effects have traditionally been thought to last only a few seconds at most, there is recent evidence that such priming can be more persistent (Becker, Moscovitch, Behrmann, \& Joordens, 1997; Hughes \& Whittlesea, 2003; Joordens \& Becker, 1997). The demonstrations of persistent semantic priming have depended on more complex priming operations and, sometimes, multiple priming events. The semantic priming effects demonstrated in the present research correspond to this evidence, in that the priming event(s) required relatively complex category identification operations and memory maintenance processes, and the observed priming was evident after an average of five intervening trial components.

The magnitude of priming effects observed in these experiments was also noteworthy. When priming effects are expressed as percentages of savings in response speed, the average priming effect for focused categories across all the experiments was approximately $10 \%$. This is substantial when one considers that this priming effect was general to previously unprocessed exemplars of a category and was moderately persistent.

It is also noteworthy that some general category priming occurred even after relatively little processing of that category. In all the experiments except Experiment 2, reliable ignored category priming resulted from minimal initial rehearsal of the exemplars prior to the memory selection instruction. Of particular interest, ignored category priming occurred in Experiment 4 when there was only one exemplar representing the ignored category. This suggests that information in LTM has increased availability for processing after relatively little attention has been devoted to semantically related information.

In interpreting the magnitude of the observed priming effects, it should be noted that these effects were probably influenced by contextual factors within the experiments. In particular, there was no attempt to mask the relationship between the content of the memory load and the subsequent category comparisons in which priming was measured. Although it is unlikely that the participants actively maintained memory load content after the recall responses, it is likely that the participants learned, either explicitly or implicitly, that there would be subsequent reference to both categories in the memory load. Prior evidence for semantic priming when the lexical decision task was used suggests that contextual variables such as the proportion of trials that are primed can influence expectations of target content, which can increase priming effects (Neely, 1977; Neely, Keefe, \& Ross, 1989). However, a variety of evidence supports the conclusion that semantic priming cannot be attributed solely to expectation or stra- 
tegic processes related to contextual variables (McNamara \& Holbrook, 2003).

Although it is likely that contextual influences contributed to the magnitude of priming in the present experiments, it also seems likely that these influences exist in everyday manifestations of ALTM. For example, during language comprehension, readers and listeners have learned from experience that information acquired early in a passage or conversation will likely be needed again soon. Such awareness might not initiate active rehearsal, but it may affect the level of availability of relevant information in the same way that context effects in priming experiments affect the magnitude of observed facilitation.

\section{Attentional Resources and ALTM}

Some evidence from these experiments suggests that ALTM following attention-driven processing operations is not constrained by the same resource limits as are attentional processes. The findings from Experiments 3 and 4 are consistent with a view either that ALTM processes are an automatic consequence of attention that require no additional resources or that they rely on different cognitive resources than do the attention processes. Such a conclusion seems most consistent with Cowan's (1999) embedded process model of WM, in which the limits to activation (i.e., time) are independent of the limits to attention (number of chunks). However, the issue of cognitive resources underlying attention and activation processes in WM is complex. In our opinion, it requires a variety of different forms of evaluation before firm conclusions can be drawn. Nevertheless, if the conclusion from the present experiments holds up in the light of further evidence, it would raise important questions as to the limits of ALTM during any processing event. As has been noted by Cowan (1999), prior evidence suggests that activation decays with time. It is possible, however, that activation might also be limited by the number of elements sharing activation (Anderson et al., 1996). These issues will require considerably more empirical evidence to resolve.

\section{Theoretical Interpretation of ALTM Effects}

The pattern of priming effects reported here is generally consistent with all of the previously discussed WM models that incorporate active or accessible LTM as part of WM (Anderson, 1983, 1993; Anderson et al., 1996; Cantor \& Engle, 1993; Cowan, 1995, 1999; Ericsson \& Kintsch, 1995; Just \& Carpenter, 1992; Oberauer, 2002). The present experiments were not designed to explicitly contrast these models; instead, they were designed to test the fundamental assumption of greater LTM availability that underlies all of them. The evidence supported the notions that memory contents that are semantically related to what has been processed in WM are made more available, the type and amount of WM processing dictates the degree of availability, and the increased availability persists through at least several intervening processing events.

An important theoretical question relevant to all the WM models is the following: What memory mechanisms are responsible for the availability of related LTM evident in the present experiments? Alternative mechanisms proposed in the WM literature correspond, to some degree, to different explanations found in the literature on semantic and conceptual priming. We will conclude this discussion by considering how the present evidence fits with these different accounts.

Activation in existing memory structures is proposed by some models to account for LTM availability in WM (e.g., Anderson, 1983, 1993; Cowan, 1995, 1999; Just \& Carpenter, 1992). In the context of the present experiments, this account would imply that attention focus on exemplars in the memory load and, in some cases, a category label produced activation that spread throughout associative links connecting representations of new category exemplars and the category concept. This activation would then underlie facilitated performance on subsequent category comparisons for the focused and ignored categories. Such an activation explanation corresponds to common interpretations of short-term semantic priming effects (McNamara, 1992).

Despite the general appeal of this explanation, the time course of the present category priming effects raises some problems. Following memory load encoding, selection, and recall, our experiments included two unrelated warmup category comparisons prior to a randomly ordered sequence of comparisons from unprimed, focused, and ignored categories. Thus, the priming effects observed in the focused and ignored categories occurred after several unrelated, intervening comparison trials between memory load processing and the target comparison trials. Becker et al. (1997) noted that a temporary spreading activation explanation cannot account easily for such long-lasting semantic priming without some additional assumptions. They proposed and found empirical support for a neural network model of semantic priming that included a persistent form of memory strengthening.

In contrast to an activation account, the present ALTM priming effects could also be explained by an account that emphasizes memory for mental operations performed in each trial. Such a procedural or skilled memory account would attribute facilitation in primed category comparisons to the strengthening of category-specific membership identification operations. These operations would be strengthened during the memory selection phase of a trial (i.e., when the participants had to identify and select exemplars of a specific category from the memory load). Then, during the subsequent category comparison phase of each trial, the recent strengthening of this category-specific procedure would facilitate related category comparisons, even if memory representations for these new category exemplars had not received lasting activation from prior category processing. This account partially corresponds to the skilled memory retrieval mechanism posited by Ericsson and Kintsch (1995) in their theory of long-term WM. It also corresponds to memory-for-operations accounts of long-term conceptual and semantic priming (Hughes \& Whittlesea, 2003; Woltz, 1996).

A procedural account accommodates the persistence of the priming effects found in these experiments more 
readily than does a traditional spreading activation account without a long-term strength component. However, it appears to be less consistent with other features of the present ALTM evidence. In several experiments, we found significant priming in categories for which no practice was provided on category exemplar identification in the memory load. In Experiment 2, half the trials presented instructions to ignore one memory load category. Consequently, the focused category was not labeled, and no category membership comparisons were required for it. Yet priming for the focused categories was equivalent to that for the ignored categories for which category membership identification was required. Moreover, in Experiment 4 , in which only select instructions were used for category identification, ignored category priming was as large as focused category priming (with a trend toward being larger) when there were three ignored category exemplars and only one focused category exemplar. In other words, encoding three category exemplars without category membership identification produced as much priming as was produced when category membership identification was required for one exemplar. One could argue that the participants implicitly, rather than explicitly, identified the category membership of the ignored category exemplars. We do not completely discount this possibility. However, we believe that this evidence is more consistent with an account that assumes some degree of activation or strengthening of general category concepts, in addition to category membership identification operations.

On the basis of the patterns of priming observed under the various trial conditions, we propose an account for ALTM that includes both the strengthening of existing lexical and semantic memory representations and the strengthening of procedural memory for mental operations. In part, this reflects the perceived limits of either single explanation. It also reflects the compelling case that has been made for acknowledging declarative and procedural memory systems that function in concert in most complex processing tasks (Anderson, 1983, 1993; Schacter \& Tulving, 1994; Schacter, Wagner, \& Buckner, 2000; Squire, 1994; Squire, Knowlton, \& Musen, 1993). ${ }^{2}$

Although we see a need to include both semantic and procedural strengthening components to account for the ALTM effects, we assume that most of the observed priming reflects procedural strengthening. We make this claim for several reasons. First, consistent with Becker et al. (1997), we reject the idea that simple spreading activation can account for the persistent priming observed in the processing of new exemplars. Persistent priming of lexical or semantic memory representations seems possible only for information directly processed in the priming events. In other words, we acknowledge the likely contribution of persistent conceptual repetition priming but discount the contribution of temporary spreading activation to our observed priming effects. In the present experiments, new, previously unseen exemplars always made up the target comparison trials. We assume that strength for their corresponding memory representations was not increased following memory load processing beyond a few seconds. We do assume that their corresponding category concept representations had some persistent increased memory strength due to repetition priming, especially if the category was labeled in the memory selection frame. However, it seems unlikely that this restricted scope of repetition priming could account for the majority of the observed priming effects.

Second, on the basis of evidence from previous conceptual and semantic priming research, we assume that a substantial amount of the observed priming effects are attributable to memory for operations performed on stimulus content, rather than to memory for the stimulus content per se (Hughes \& Whittlesea, 2003; Woltz, 1996). In this case, memory for category membership identification processes performed in the initial phase of each trial is assumed to facilitate any subsequent comparison trial that requires the same or a similar operation. Memory for such operations has been shown to be category specific but general to different exemplars of a category (Hughes \& Whittlesea, 2003). It has also been shown to account for the majority of priming effects and to be relatively persistent (Hughes \& Whittlesea, 2003; Woltz, 1996). In our view, viable explanations of ALTM must include some mechanism for the influence of repeated mental operations, although in most cases, this is likely to be combined with persistent conceptual repetition priming of stimulus content.

\section{REFERENCES}

Anderson, J. R. (1983). The architecture of cognition. Cambridge, MA: Harvard University Press.

Anderson, J. R. (1993). Rules of the mind. Hillsdale, NJ: Erlbaum.

Anderson, J. R., Reder, L. M., \& Lebiere, C. (1996). Working memory: Activation limitations on retrieval. Cognitive Psychology, 30, 221-256.

BAdDEley, A. D. (1986). Working memory. Oxford: Oxford University Press.

BADDELEY, A. [D.] (1993). Working memory or working attention? In A. Baddeley \& L. Weiskrantz (Eds.), Attention: Selection, awareness, and control. A tribute to Donald Broadbent (pp. 152-170). Oxford: Oxford University Press, Clarendon Press.

BADDELEY, A. [D.] (2000). The episodic buffer: A new component of working memory? Trends in Cognitive Sciences, 4, 417-423.

BADDELEY, A. [D.] (2001). Is working memory still working? American Psychologist, 56, 849-864.

BadDEley, A. D., \& Hitch, G. J. (1974). Working memory. In G. Bower (Ed.), The psychology of learning and motivation (Vol. 8, pp. 47-90). San Diego: Academic Press.

Battig, W. F., \& Montague, W. E. (1969). Category norms for verbal items in 56 categories: A replication and extension of the Connecticut category norms. Journal of Experimental Psychology Monographs, 80(3, Pt. 2), 1-46.

Becker, S., Moscovitch, M., Behrmann, M., \& Joordens, S. (1997). Long-term semantic priming: A computational account and empirical evidence. Journal of Experimental Psychology: Learning, Memory, \& Cognition, 23, 1059-1082.

Broadbent, D. E. (1993). Comparison with human experiments. In D. E. Broadbent (Ed.), The simulation of human intelligence (pp. 198217). Oxford: Blackwell.

CAntor, J., \& EngLe, R. W. (1993). Working-memory capacity as longterm memory activation: An individual-differences approach. Journal of Experimental Psychology: Learning, Memory, \& Cognition, 19, 1101-1114.

Cowan, N. (1995). Attention and memory: An integrated framework. New York: Oxford University Press. 
CowAN, N. (1999). An embedded-processes model of working memory. In A. Miyake \& P. Shah (Eds.), Models of working memory: Mechanisms of active maintenance and executive control (pp. 62-101). New York: Cambridge University Press.

Daneman, M., \& CARPEnter, P. A. (1980). Individual differences in working memory and reading. Journal of Verbal Learning \& Verbal Behavior, 19, 450-466.

ERICSSON, K. A., \& Kintsch, W. (1995). Long-term working memory. Psychological Review, 102, 211-245.

GERNSBACHER, M. A. (1990). Language comprehension as structure building. Hillsdale, NJ: Erlbaum.

Haarmann, H. J., Davelaar, E. J., \& Usher, M. (2003). Individual differences in semantic short-term memory capacity and reading comprehension. Journal of Memory \& Language, 48, 320-345.

Hughes, A. D., \& Whittlesea, B. W. A. (2003). Long-term semantic transfer: An overlapping-operations account. Memory \& Cognition, 31, 401-411.

JoORDENS, S., \& BECKER, S. (1997). The long and short of semantic priming effects in lexical decision. Journal of Experimental Psychology: Learning, Memory, \& Cognition, 23, 1083-1105.

Just, M. A., \& CARPenter, P. A. (1992). A capacity theory of comprehension: Individual differences in working memory. Psychological Review, 99, 122-149.

KInTsCH, W. (1998). Comprehension: A paradigm for cognition. New York: Cambridge University Press.

Kintsch, W., PAtel, V. L., \& ERICsSON, K. A. (1999). The role of longterm working memory in text comprehension. Psychologia, 42, 186-198.

McNamara, T. P. (1992). Priming and constraints it places on theories of memory and retrieval. Psychological Review, 99, 650-662.

McNamara, T. P., \& Holbrook, J. B. (2003). Semantic memory and priming. In I. B. Weiner (Series Ed.) \& R. W. Proctor \& A. F. Healy (Vol. Eds.), Handbook of psychology: Vol. 4. Experimental psychology (pp. 447-474). New York: Wiley.

MiYake, A., \& Shah, P. (1999). Models of working memory: Mechanisms of active maintenance and executive control. New York: Cambridge University Press.

NEELY, J. H. (1977). Semantic priming and retrieval from lexical memory: Roles of inhibitionless spreading activation and limited-capacity attention. Journal of Experimental Psychology: General, 106, 226-254.

Neely, J. H., Keefe, D. E., \& Ross, K. L. (1989). Semantic priming in the lexical decision task: Roles of prospective prime-generated expectancies and retrospective semantic matching. Journal of Experimental Psychology: Learning, Memory, \& Cognition, 15, 1003-1019.

OBerauer, K. (2002). Access to information in working memory: Exploring the focus of attention. Journal of Experimental Psychology: Learning, Memory, \& Cognition, 28, 411-421.

Potter, M. C. (1993). Very short-term conceptual memory. Memory \& Cognition, 21, 156-161.

SCHACTER, D. L., \& Tulving, E. (1994). What are the memory systems of 1994? In D. L. Schacter \& E. Tulving (Eds.), Memory systems 1994 (pp. 1-38). Cambridge, MA: MIT Press.

Schacter, D. L., Wagner, A. D., \& Buckner, R. L. (2000). Memory systems of 1999. In E. Tulving \& F. I. M. Craik (Eds.), The Oxford handbook of memory (pp. 627-643). New York: Oxford University Press.

Schneider, W., Eschman, A., \& Zuccolotto, A. (2002). E-Prime user's guide (Version 1.1). Pittsburgh: Psychology Software Tools.

SQuire, L. R. (1994). Declarative and nondeclarative memory: Multiple brain systems supporting learning and memory. In D. L. Schacter \& E. Tulving (Eds.), Memory systems 1994 (pp. 203-231). Cambridge, MA: MIT Press.

Squire, L. R., Knowlton, B., \& Musen, G. (1993). The structure and organization of memory. Annual Review of Psychology, 44, 453-495.

Woltz, D. J. (1990). Repetition of semantic comparisons: Temporary and persistent priming effects. Journal of Experimental Psychology: Learning, Memory, \& Cognition, 16, 392-403.

Woltz, D. J. (1996). Perceptual and conceptual priming in a semantic reprocessing task. Memory \& Cognition, 24, 429-440.

\section{NOTES}

1. Recently, Baddeley $(2000,2001)$ proposed a substantial addition to his model with the inclusion of a separate multimodal storage buffer that holds integrated episodic representations.

2. Different theorists debate the issues of what constitutes a memory system, what terminology best describes important memory distinctions, and even what distinguishes some of the proposed systems. However, some form of distinction between declarative (or episodic and semantic) and procedural (or implicit or nondeclarative) memories or processes is made by most.

\begin{tabular}{|c|c|c|c|c|c|c|c|}
\hline \multicolumn{8}{|c|}{$\begin{array}{c}\text { APPENDIX } \\
\text { Stimulus Sets }\end{array}$} \\
\hline Set & Category Label & $\begin{array}{l}\text { Memory } \\
\text { Word } 1\end{array}$ & $\begin{array}{l}\text { Memory } \\
\text { Word } 2\end{array}$ & $\begin{array}{c}\text { Positive Match } \\
\text { Word } 1\end{array}$ & $\begin{array}{c}\text { Positive Match } \\
\text { Word } 2\end{array}$ & $\begin{array}{c}\text { Negative } \\
\text { Match } \\
\text { Word } 1\end{array}$ & $\begin{array}{c}\text { Negative } \\
\text { Match } \\
\text { Word } 2\end{array}$ \\
\hline 1 & Precious stone & ruby & diamond & emerald & sapphire & opal & snail \\
\hline 1 & Unit of time & decade & minute & century & month & hour & fudge \\
\hline 1 & Relative & daughter & uncle & aunt & brother & mother & soil \\
\hline 2 & Distance & inch & kilometer & mile & foot & centimeter & chain \\
\hline 2 & Metal & silver & bronze & iron & copper & aluminum & feathers \\
\hline 2 & Reading material & book & magazine & newspaper & novel & pamphlet & zipper \\
\hline 3 & Military rank & captain & sergeant & lieutenant & general & colonel & hoe \\
\hline 3 & Animal & $\operatorname{dog}$ & elephant & horse & lion & cat & garbage \\
\hline 3 & Kind of cloth & cotton & rayon & wool & silk & nylon & frame \\
\hline 4 & Color & yellow & purple & blue & red & green & hungry \\
\hline 4 & Kitchen utensil & fork & spatula & spoon & knife & pan & hug \\
\hline 4 & Religious building & temple & chapel & church & synagogue & cathedral & blade \\
\hline 5 & Part of speech & adverb & pronoun & noun & adjective & verb & memory \\
\hline 5 & Piece of furniture & table & sofa & chair & bed & desk & pasta \\
\hline 5 & Body part & stomach & finger & legs & head & arms & sidewalk \\
\hline 6 & Fruit & apple & cherry & pear & banana & peach & wedding \\
\hline 6 & Weapon & rifle & missile & sword & bomb & cannon & eraser \\
\hline 6 & Political office & mayor & sheriff & president & senator & governor & main street \\
\hline 7 & Dwelling & cottage & mansion & house & apartment & shack & candle \\
\hline 7 & Alcoholic drink & whiskey & brandy & vodka & beer & wine & cradle \\
\hline 7 & Country & Russia & England & Italy & Japan & Canada & treasury \\
\hline 8 & Crime & murder & arson & robbery & assault & embezzling & cooking \\
\hline
\end{tabular}


APPENDIX (Continued)

\begin{tabular}{|c|c|c|c|c|c|c|c|}
\hline Set & Category Label & $\begin{array}{l}\text { Memory } \\
\text { Word } 1\end{array}$ & $\begin{array}{l}\text { Memory } \\
\text { Word } 2\end{array}$ & $\begin{array}{c}\text { Positive Match } \\
\text { Word } 1\end{array}$ & $\begin{array}{c}\text { Positive Match } \\
\text { Word } 2\end{array}$ & $\begin{array}{c}\text { Negative } \\
\text { Match } \\
\text { Word } 1\end{array}$ & $\begin{array}{c}\text { Negative } \\
\text { Match } \\
\text { Word } 2\end{array}$ \\
\hline 8 & Carpentry tool & hammer & chisel & screwdriver & wrench & pliers & chalkboard \\
\hline 8 & Clergy member & rabbi & preacher & priest & bishop & minister & whale \\
\hline 9 & Seasoning & pepper & garlic & vanilla & salt & cinnamon & paper \\
\hline 9 & Fuel & coal & kerosene & diesel & propane & gasoline & humor \\
\hline 9 & Occupation & dentist & plumber & lawyer & engineer & doctor & airplane \\
\hline 10 & Geological feature & mountain & river & valley & volcano & plateau & mildew \\
\hline 10 & Sport & football & tennis & baseball & golf & soccer & ladder \\
\hline 10 & Weather phenomenon & rain & tornado & snow & hurricane & wind & letters \\
\hline 11 & Piece of clothing & jacket & trousers & dress & sweater & blouse & crumb \\
\hline 11 & Part of a building & window & ceiling & door & wall & floor & figure \\
\hline 11 & Chemical element & carbon & sulfur & hydrogen & sodium & helium & blister \\
\hline 12 & Musical instrument & trumpet & guitar & piano & violin & clarinet & pillow \\
\hline 12 & Kind of money & dollar & nickel & dime & penny & quarter & glue \\
\hline 12 & Bird & robin & eagle & sparrow & blue jay & canary & triangle \\
\hline 13 & Type of music & jazz & classical & rock ' $\mathrm{n}$ ' roll & opera & symphony & cement \\
\hline 13 & Beverage & coffee & soda & milk & tea & lemonade & fertilizer \\
\hline 13 & Car & Chevy & Honda & Ford & Toyota & Chrysler & wonder \\
\hline 14 & Science & physics & botany & chemistry & biology & astronomy & humidity \\
\hline 14 & Toy & puzzle & marbles & doll & jacks & teddy bear & filter \\
\hline 14 & Dance & tango & ballet & waltz & polka & cha-cha & Morse code \\
\hline 15 & Vegetable & carrot & spinach & tomato & bean & lettuce & pebble \\
\hline 15 & Type of footwear & sandal & slipper & shoe & boot & moccasins & shelves \\
\hline 15 & Insect & spider & beetle & ant & mosquito & bee & flour \\
\hline 16 & Girl's name & Linda & Mary & Ann & Jane & Barbara & Chancellor \\
\hline 16 & Flower & tulip & orchid & rose & carnation & daisy & envelope \\
\hline 16 & Disease & cancer & measles & tuberculosis & polio & smallpox & blonde \\
\hline 17 & Tree & cedar & maple & oak & pine & elm & clay \\
\hline 17 & Boy's name & Richard & Charles & John & Bob & Bill & Pepsi \\
\hline 17 & Watercraft & sailboat & steamship & yacht & canoe & submarine & chapter \\
\hline 18 & Fish & catfish & salmon & trout & shark & tuna & parade \\
\hline 18 & City & New York & London & Chicago & Baltimore & Paris & Sears \\
\hline 18 & Emotion & happy & jealous & angry & envious & embarrassed & clean \\
\hline 19 & Snake & cobra & rattler & python & boa & garter & opaque \\
\hline 19 & State & Texas & Georgia & California & Florida & Pennsylvania & publisher \\
\hline 19 & Meal & breakfast & supper & dinner & lunch & brunch & shadow \\
\hline 20 & College & Harvard & Purdue & Stanford & Penn State & Yale & Wendy's \\
\hline 20 & Royal title & duchess & princess & king & prince & queen & knot \\
\hline 20 & Car part & piston & axle & carburetor & transmission & cylinder & trail \\
\hline 21 & Planet & Venus & Saturn & Mars & Mercury & Jupiter & Rose Bowl \\
\hline 21 & Room & kitchen & hallway & bathroom & den & basement & cough \\
\hline 21 & Stage of life & infant & adult & adolescent & newborn & elderly & jam \\
\hline 22 & Level of education & grade school & university & junior high & post graduate & college & winter \\
\hline 22 & Eating establishment & cafe & drive-in & restaurant & fast-food & diner & mower \\
\hline 22 & Continent & Asia & Europe & Africa & North America & Australia & Kmart \\
\hline 23 & Form of transportation & airplane & subway & automobile & train & bus & classroom \\
\hline 23 & Dessert & pudding & cookie & cake & pie & ice cream & noise \\
\hline 23 & Religious holiday & Christmas & Hanukkah & Easter & Passover & Good Friday & Karl Marx \\
\hline 24 & Breakfast cereal & Wheaties & Corn Flakes & Cheerios & Quaker Oats & Chex & comet \\
\hline 24 & Branch of the military & Air Force & Marines & Army & Navy & Coast Guard & salesmen \\
\hline 24 & Type of vehicle & pickup & sedan & station wagon & sports car & van & radio \\
\hline
\end{tabular}

(Manuscript received March 23, 2004;

revision accepted for publication April 30, 2005.) 